\title{
Order-Disorder Transition in the Half-Filled Two-Component Lattice Fermion Model with Nearest-Neighbor Repulsion
}

\author{
Konrad Jerzy Kapcia ${ }^{1}$ (D) - Katarzyna Majewska-Albrzykowska²
}

Received: 7 November 2019 / Accepted: 1 February 2020 / Published online: 14 March 2020

(C) The Author(s) 2020

\begin{abstract}
In this work, we study a model of asymmetric two-component lattice fermion system at half-filling, where particles of both species (distinguishable by, e.g., spin) can interact only via nearest-neighbor repulsion $W$. The analysis is performed on the Bethe lattice using the Hartree-Fock-type mean-field approximation, which is rigorous in the limit of high dimensions. At sufficiently low temperatures, both antiferromagnetic and charge orders (related to inhomogeneous distribution of particles with both spins) coexist in the system. We find that an order-disorder continuous transition occurs with increasing temperature. The transition temperature depends on the ratio $t_{\uparrow} / t_{\downarrow}$ of the hopping amplitudes of both fermion species (i.e., the asymmetry of the model). For fixed $W$, it is the biggest if one component is localized (e.g., $t_{\uparrow}=0$ ), and it decreases to its minimal value for the same hopping amplitudes $\left(t_{\uparrow}=t_{\downarrow}\right)$. Moreover, it increases with $W$ for fixed $t_{\uparrow} / t_{\downarrow}$. Dependencies of order parameters with model parameters and temperature are also presented.
\end{abstract}

Keywords Order-disorder transition · Two-component fermion model $\cdot$ Intersite repulsion · Mean-field theory · Charge and spin orders $\cdot$ Fermionic gases

\section{Introduction}

The physics of strongly correlated fermion (electron) systems is still very intensively studied topic. These systems exhibit quite complex phase diagrams with a variety of very interesting phenomena such as metal-insulator transitions and superconductivity as well as a competition between various ordered phases, e.g., [1-5]. Understanding them is important not only in the context of solid state physics, but also for physics of quantum gases, where intensive experimental progress occurs recently (for a review see, e.g., [6-10]). In such systems, for example, various inter-particle interactions can be tuned precisely.

In this paper, we study theoretically a very special system, namely the asymmetric two-component fermionic

Konrad Jerzy Kapcia

konrad.kapcia@ifj.edu.pl

1 Institute of Nuclear Physics, Polish Academy of Sciences, ul. W. E. Radzikowskiego 152, PL-31342 Kraków, Poland

2 Faculty of Physics and Astronomy, University of Wrocław, Plac Maxa Borna 9, PL-50204 Wrocław, Poland
Hamiltonian on the lattice, where itinerant particles of both species (distinguishable, e.g., by the spin index $\sigma \in\{\uparrow, \downarrow\}$ ) can interact only via intersite Coulomb interactions restricted to the nearest neighbors (NNs). The main aim is to analyze the effects of a change of the relative ratio between two fermion species hopping amplitudes (i.e., the asymmetry of the model) on both charge and spin orders at the half-filling. Thus, we neglect onsite Hubbard $U$ interaction here (i.e., $U=0$ ). In particular, we investigate a dependence of the continuous (second order) order-disorder transition temperature on the model parameters.

The paper is organized as follows. In Section 2, the explicit form of the model investigated is shown, whereas Section 3 includes equations obtained within the mean-field approximation. Section 4 is devoted to numerical results, where the ground state properties (Section 4.1) and behavior in finite temperature (Sections 4.2 and 4.3) are discussed. The conclusions are included in Section 5 .

\section{The Model}

The Hamiltonian of the studied system (on the infinite lattice) consists of three terms (the kinetic term, the 
intersite interaction term, and the chemical potential term, respectively) and it has the following form:

$\hat{H}=\sum_{\langle i, j\rangle, \sigma} \frac{t_{\sigma}}{\sqrt{z}} \hat{c}_{i, \sigma}^{\dagger} \hat{c}_{j, \sigma}+\frac{W}{2 z} \sum_{\langle i, j\rangle} \hat{n}_{i} \hat{n}_{j}-\mu \sum_{i, \sigma} \hat{n}_{i, \sigma}$,

where $\hat{c}_{i, \sigma}^{\dagger}\left(\hat{c}_{i, \sigma}\right)$ is an operator of creation (annihilation) of fermionic particle (electron) with spin $\sigma \in \uparrow, \downarrow$ at site $i$, $\hat{n}_{i}=\sum_{\sigma} \hat{n}_{i, \sigma}=\hat{n}_{i, \uparrow}+\hat{n}_{i, \downarrow}$ is the number of particles at site $i$, and $\hat{n}_{i, \sigma}=\hat{c}_{i, \sigma}^{\dagger} \hat{c}_{i, \sigma}$ is number of particles with spin $\sigma$ at site $i . \sum_{\langle i, j\rangle}$ denotes the summation over NNs, independently. $t_{\sigma}$ is the hopping between NNs for particles with spin $\sigma, W$ is the intersite density-density interaction between NNs, and $\mu$ denotes the chemical potential. The scaling of the model parameters with $z$, i.e., with the number of NNs, is so chosen to obtain the finite energy in $z \rightarrow \infty$ limit [11-14].

In the following, we restrict ourselves to the case of the intersite repulsion (i.e., $W>0$ ). We will consider model (1) on the Bethe lattice with a large coordination number ( $z \rightarrow \infty$ limit), for which the (non-interacting) density of states for each $\sigma$ is semi-elliptic:

$\rho_{\sigma}(\epsilon)=\frac{2}{\pi D_{\sigma}} \sqrt{1-\left(\epsilon / D_{\sigma}\right)^{2}}$ for $|\epsilon|<D_{\sigma}$

and $\rho_{\sigma}(\epsilon)=0$ for $|\epsilon|>D_{\sigma}$, where $D_{\sigma}=2 t_{\sigma}$ is the half-bandwidth for electrons with spin $\sigma$ [2]. Moreover, we investigate the model at the half-filling, i.e., for $n=1$, where $n=\left\langle\sum_{i} \hat{n}_{i}\right\rangle / L$ ( $L$ is the total number of sites and $\langle\hat{A}\rangle$ is the thermodynamic average of operator $\hat{A}$ ). It is equivalent to $\mu=W$ condition, which is a rigorous statement for any bipartite (alternate) lattice for model (1) and $n_{\sigma}=1 / 2$ for both $\sigma$ in this case (where $n_{\sigma}=$ $\left.\left\langle\sum_{i} \hat{n}_{i, \sigma}\right\rangle / L\right)$.

Note that the model considered is equivalent to the $U=0$ asymmetric extended Hubbard model (for $W=0$ case see [15-17]). In particular, for $t_{\uparrow}=t_{\downarrow}$, it corresponds to the $U=0$ extended Hubbard model (e.g., [18-23] and references therein) or to the spinless fermion model [24, 25], whereas, for $t_{\uparrow}=0$ and $t_{\downarrow} \neq 0$, it reduces to the $U=0$ extended Falicov-Kimball model [26-31]. In this work, a general case of any $t_{\uparrow} \geq 0$ and $t_{\downarrow} \geq 0$ (with restriction $t_{\uparrow} \geq t_{\downarrow}$ ) will be considered. For the case of $t_{\uparrow} \leq t_{\downarrow}$, the finding of the work remains unchanged with only a change of the sign of staggered magnetization $M_{Q}$ (defined in the next section).

\section{The Method}

The alternate lattice is such a lattice, which can be divided into two equivalent sublattices, e.g., $A$ and $B$ sublattices (the Bethe lattice has this feature) [1-3]. In this paper, we consider only two-sublattice ordering (of checker-board type). It means that an average of any operator calculated at site $i$ belonging to sublattice $\alpha$ ( $i \in \alpha$, where $\alpha=A, B$ ) will be the same. In particular, let us define the concentration of particles with spin $\sigma$ on sublattice $\alpha: n_{\alpha, \sigma} \equiv\left\langle\hat{n}_{i, \sigma}\right\rangle$ for any $i \in \alpha$ (with such a definition, one can also write $\left.n_{\alpha, \sigma}=(2 / L) \sum_{i \in \alpha}\left\langle\hat{n}_{\alpha, \sigma}\right\rangle\right)$.

The thermodynamic average of operator $\hat{A}$ is defined as $\langle\hat{A}\rangle=\operatorname{Tr}[\exp (-\beta \hat{H}) \hat{A}] / Z$, where $Z=\operatorname{Tr}[\exp (-\beta \hat{H})]$ is the grand sum of states (the partition function), $1 / \beta=k_{B} T$, $T$ is the temperature, and $k_{B}$ is the Boltzmann constant. $\operatorname{Tr} \hat{B}$ is the trace of any operator $\hat{B}$, and it is calculated in the Fock space (in the grand canonical ensemble) here.

Using the mean-field broken-symmetry Hartree-Fock approximation (restricting ourselves only to the Hartree terms in the intersite term decoupling) and performing the Bogoliubov transformation to Hamiltonian (1) at the halffilling ( $\mu=W, n=1)$, one can derive the free energy $F=\Omega+\mu n$ per site (where $\Omega=-1 /(L \beta) \ln Z$ is the grand canonical potential and $Z$, defined above, is calculated by using the mean-field Hamiltonian, $T>0$ ). After straightforward, but rather lengthy, calculation, one gets

$F=(W / 2)\left(\Delta_{Q}^{2}+1\right)-\left(K_{\uparrow}+K_{\downarrow}\right)$,

where

$K_{\sigma}=\frac{1}{\beta} \int \rho_{\sigma}(\epsilon) \ln \left[2 \cosh \left(\frac{\beta E(\epsilon)}{2}\right)\right] d \epsilon$,

and $E(\epsilon)=\sqrt{\epsilon^{2}+W^{2} \Delta_{Q}^{2}}(\sigma \in\{\uparrow, \downarrow\}$ as previously). All integrals in this work are calculated in $\left[-D_{\sigma}, D_{\sigma}\right]$ interval, practically.

One can also obtain the following equations for the charge polarization $\Delta_{Q} \equiv\left[\left(n_{A, \uparrow}+n_{A, \downarrow}\right)-\left(n_{B, \uparrow}+\right.\right.$ $n_{B, \downarrow}$ )]/2 (difference between total concentrations in both sublattices, associated with inhomogeneous distribution of particles) and the staggered magnetization $M_{Q} \equiv\left[\left(n_{A, \uparrow}-\right.\right.$ $\left.\left.n_{A, \downarrow}\right)-\left(n_{B, \uparrow}-n_{B, \downarrow}\right)\right] / 2$ (difference between total magnetic moments in both sublattices, if $\sigma$ is real spin):

$$
\begin{gathered}
\Delta_{Q}\left[2-W\left(B_{\uparrow}+B_{\downarrow}\right)\right]=0, \\
M_{Q}=\frac{1}{2} W \Delta_{Q}\left(B_{\uparrow}-B_{\downarrow}\right),
\end{gathered}
$$

where

$B_{\sigma}=\int \frac{\rho_{\sigma}(\epsilon)}{E(\epsilon)} \tanh \left[\frac{\beta E(\epsilon)}{2}\right] d \epsilon . \quad\left(W \Delta_{Q} \neq 0\right)$

Note that the right side of (6) is not a function of $M_{Q}$ and the problem is reduced to solving single non-linear (5). The expression in the brackets in the left side of (5) is associated with the nontrivial solution $\Delta_{Q} \neq 0$. For $\Delta_{Q}=0$, (6) gives $M_{Q}=0$. Moreover, (5) is equivalent to the condition $\partial F / \partial \Delta_{Q}=0$. Due to equivalence of two sublattices, we can restrict ourselves to the solutions with $\Delta_{Q} \geq 0$. Note also that for $t_{\uparrow} \leq t_{\downarrow}$ one gets $M_{Q} \geq 0$. 
Fig. 1 Parameters $\Delta_{Q}$ (a) and $M_{Q}(\mathbf{b})$ as a function of $W / t_{\downarrow}$ for a few values of $t_{\uparrow} / t_{\downarrow}$ (from the top: $0.00,0.01,0.05,0.10$, $0.30,0.60,1.00)$ in the ground state

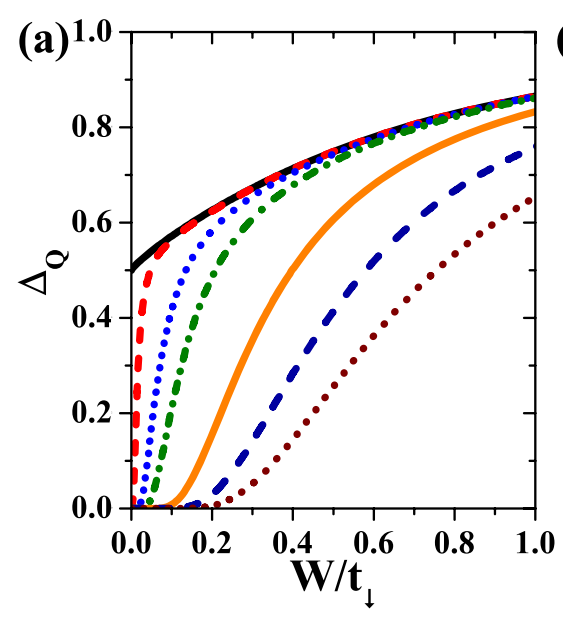

Assuming that $\Delta_{Q} \rightarrow 0$ (but $\Delta_{Q} \neq 0$ ) if $T \rightarrow T_{C}$ (obviously $M_{Q} \rightarrow 0$ simultaneously), one gets from (5) the following condition for temperature $T_{c}$ of continuous order-disorder transition:

$W\left(C_{\uparrow}+C_{\downarrow}\right)=2$,

where

$C_{\sigma}=\int \frac{\rho_{\sigma}(\epsilon)}{\epsilon} \tanh \left(\frac{\beta_{c} \epsilon}{2}\right) d \epsilon$

and $\beta_{c}^{-1}=k_{B} T_{c}$. For $\epsilon=0$, the expression under the integral is undetermined but the integral exists (in the sense of the Cauchy principal value). The same remark applies also to (7) for $W \Delta_{Q}=0$. As one can see in Section 4.3, all transitions for the model investigated at the half-filling are indeed continuous. Note that nonzero solution of (8) can exist only if $W>0$.

If $t_{\sigma} \rightarrow 0$ (the limit of localized electrons with spin $\sigma$; the atomic limit, cf. [32-38] and references therein), density of states $\rho_{\sigma}(\epsilon)$ approaches to the Dirac delta function $\delta(\epsilon)$ and (4), (7), and (9) get the following limiting forms, respectively:

$K_{\sigma}^{0}=(1 / \beta) \ln \left[2 \cosh \left(\beta W \Delta_{Q} / 2\right)\right]$,

$B_{\sigma}^{0}=\tanh \left(\beta W \Delta_{Q} / 2\right) /\left(W \Delta_{Q}\right), \quad\left(W \Delta_{Q} \neq 0\right)$

$C_{\sigma}^{0}=\frac{1}{2} \beta_{c}$.

Let us underline that all expressions presented in this section [(3)-(12)] are derived for the half-filling condition (i.e., $\mu=W$ ). Moreover, for $\mu=W$, one gets that total magnetization $M \equiv\left[\left(n_{A, \uparrow}-n_{A, \downarrow}\right)+\left(n_{B, \uparrow}-n_{B, \downarrow}\right)\right] / 2$ is equal to 0 and, obviously, $n \equiv\left[\left(n_{A, \uparrow}+n_{A, \downarrow}\right)+\left(n_{B, \uparrow}+\right.\right.$ $\left.\left.n_{B, \downarrow}\right)\right] / 2$ is equal to 1 for any other parameters of model (1) and temperature.

\section{Numerical Results at Half-Filling $(n=1)$}

\subsection{Ground State Properties $(T=0)$}

One can obtain the limiting expressions of (4) and (7) as well as (10) and (11) for $T=0$ (i.e., $\beta \rightarrow \infty$ ). They take the
Fig. 2 The ground state parameters $\Delta_{Q} / \Delta_{Q}^{\max }$ (a) and $M_{Q} / M_{Q}^{\max }(\mathbf{b})$ as a function of $t_{\uparrow} / t_{\downarrow}$ for a few values of $W / t_{\downarrow}$ (from the bottom-left: 0.02, 0.04, 0.06, 0.10, 0.14, 0.20, 0.30, $0.40,0.60,0.80,1.00,1.40,2.00$, and 4.00). $\Delta_{Q}^{\max } \equiv \Delta_{Q}\left(t_{\uparrow}=0\right)$ and $M_{Q}^{\max } \equiv M_{Q}\left(t_{\uparrow}=0\right)$
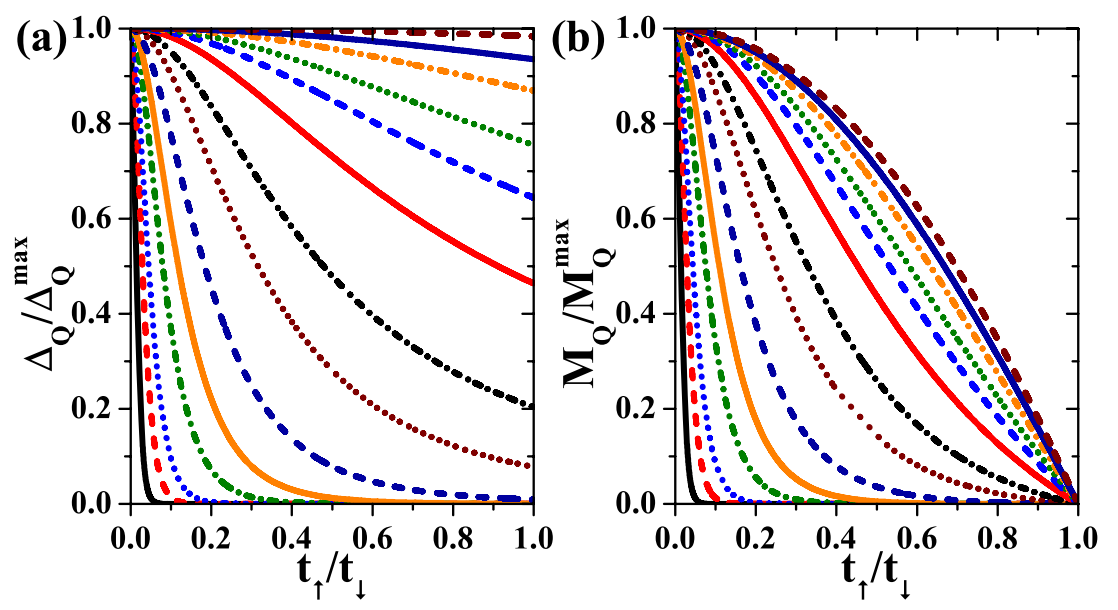
Fig. 3 Temperature $k_{B} T_{c} / W$ of order-disorder transition as a function of $W / t_{\downarrow}$ (a) and $t_{\downarrow} / W$ (b) for different ratios $t_{\uparrow} / t_{\downarrow}$ (as labeled, decreasing from the top). Values on the horizontal axes of panel (b) are so chosen to probe all space of $W$ and $t_{\downarrow}$ parameters
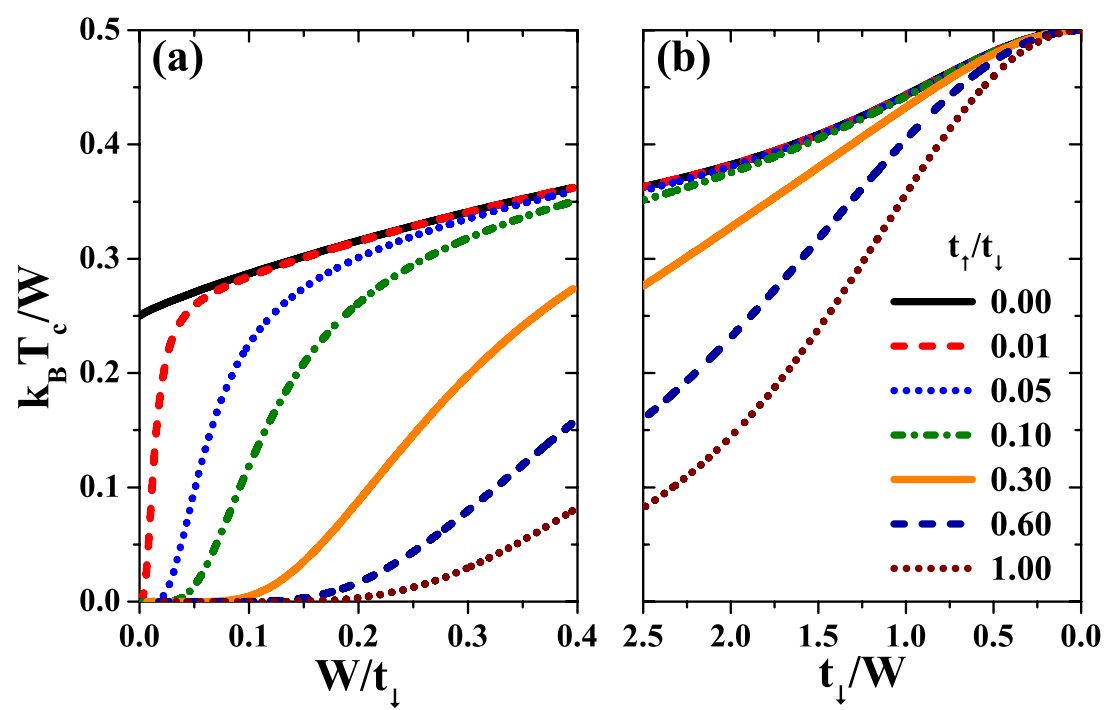

following forms, respectively: $\bar{K}_{\sigma}=(1 / 2) \int \rho_{\sigma}(\epsilon) E(\epsilon) d \epsilon$, $\bar{B}_{\sigma}=\int \rho_{\sigma}(\epsilon) / E(\epsilon) d \epsilon\left(\right.$ for $\left.t_{\sigma} \neq 0\right)$; and $\bar{K}_{\sigma}^{0}=W \Delta_{Q} / 2$, $\bar{B}_{\sigma}^{0}=1 /\left(W \Delta_{Q}\right)\left(\right.$ for $\left.t_{\sigma}=0\right)$.

In Fig. 1, the dependence of $\Delta_{Q}$ and $M_{Q}$ at the ground state as a function of $W / t_{\downarrow}$ is presented. $\Delta_{Q}$ increases with $W / t_{\downarrow}$ from 0 to its maximal value 1 at $W / t_{\downarrow} \rightarrow+\infty$, as shown in Fig. 1a (cf. [32-37] for large $W$ limit). For $t_{\uparrow} / t_{\downarrow} \neq 0$, parameter $\Delta_{Q}$ is continuous function of $W / t_{\downarrow}$, but for $t_{\uparrow} / t_{\downarrow}=0$ it exhibits a discontinuous jump from 0 at $W / t_{\downarrow}=0$ to 0.5 for $W / t_{\downarrow} \rightarrow 0^{+}$. The dependence of $M_{Q}$ as a function of $W / t_{\downarrow}$ is nonmonotonous (cf. Fig. 1b). It increases from 0 at $W / t_{\downarrow}=0$ to its maximum at some finite $W / t_{\downarrow}$, and next, it decreases to 0 for $W / t_{\downarrow} \rightarrow+\infty$. Only for $t_{\uparrow} / t_{\downarrow}=0$, it exhibits a discontinuous jump from 0 at $W / t_{\downarrow}=0$ to maximal value 0.5 for $W / t_{\downarrow} \rightarrow 0^{+}$, and then $M_{Q}$ decreases monotonously to 0 at $W / t_{\downarrow} \rightarrow+\infty$. It is clearly seen that both $\Delta_{Q}$ and $M_{Q}$ are decreasing functions of $t_{\uparrow} / t_{\downarrow}$ for fixed $W / t_{\downarrow} . \Delta_{Q}$ and $M_{Q}$ are maximal for $t_{\uparrow} / t_{\downarrow}=0$ and reach their minima for $t_{\uparrow}=t_{\downarrow}$.

Figure 2 presents $\Delta_{Q}$ and $M_{Q}$ (normalized to their values $\Delta_{Q}^{\max }$ and $M_{Q}^{\max }$ at $\left.t_{\uparrow} / t_{\downarrow}=0\right)$ as a function of $t_{\uparrow} / t_{\downarrow}$ for fixed $W / t_{\downarrow}$. For large $W / t_{\downarrow}$, parameter $\Delta_{Q}$ is almost independent on $t_{\uparrow} / t_{\downarrow}$, but for smaller $W / t_{\downarrow}$ its decreasing dependence is clearly visible. $M_{Q}$ decreases to 0 only at $t_{\uparrow}=t_{\downarrow}$ for any $W / t_{\downarrow}$. Both parameters vanishes faster (with increasing of $t_{\uparrow} / t_{\downarrow}$ ) for smaller $W / t_{\downarrow}$.

\subsection{Critical Temperature of the Continuous (Second Order) Order-Disorder Transition}

The dependence of order-disorder transition temperature $k_{B} T_{c} / W$ (determined from (8) and (9)) as a function of $W / t_{\downarrow}$ (or $t_{\downarrow} / W$ ) is shown in Fig. 3. At transition temperature $T_{c}$, both charge and antiferromagnetic orders vanish simultaneously (cf. Section 4.3). $k_{B} T_{c} / W$ is increasing function of $W / t_{\downarrow}$ (for fixed $t_{\uparrow} / t_{\downarrow}$ ) and it decreases with increasing $t_{\uparrow} / t_{\downarrow}$ (for fixed $W / t_{\downarrow}$ ). For any ratio $t_{\uparrow} / t_{\downarrow}$, temperature $k_{B} T_{c} / W$ approaches to its maximal value $1 / 2$ if $W / t_{\downarrow} \rightarrow+\infty$, reproducing the result for the atomic limit $[32,35,36]$. In the limit of $W \rightarrow 0^{+}$, one gets $T_{c} \rightarrow 0$ for any $t_{\uparrow} / t_{\downarrow}$, but $k_{B} T_{c} / W \rightarrow 0$ for $t_{\uparrow} / t_{\downarrow} \neq 0$, and $k_{B} T_{c} / W \rightarrow 1 / 4$ for $t_{\uparrow} / t_{\downarrow}=0 . T_{c}$ is a decreasing function of $t_{\uparrow} / t_{\downarrow}$ and its decrease is faster for lower values of $W / t_{\downarrow}$, cf. Fig. 4.

\subsection{Behavior of Thermodynamic Quantities $(T>0)$}

A character of the temperature dependence of parameters $\Delta_{Q}$ and $M_{Q}$ is not dependent on $W / t_{\downarrow}$ and $t_{\uparrow} / t_{\downarrow}$. Both order parameters are decreasing function of $T$ and both vanish continuously to zero at the same temperature $T_{c}$ (which coincides with the solution of (8)) as expected for continuous (second order) transitions. In Fig. 5, we show

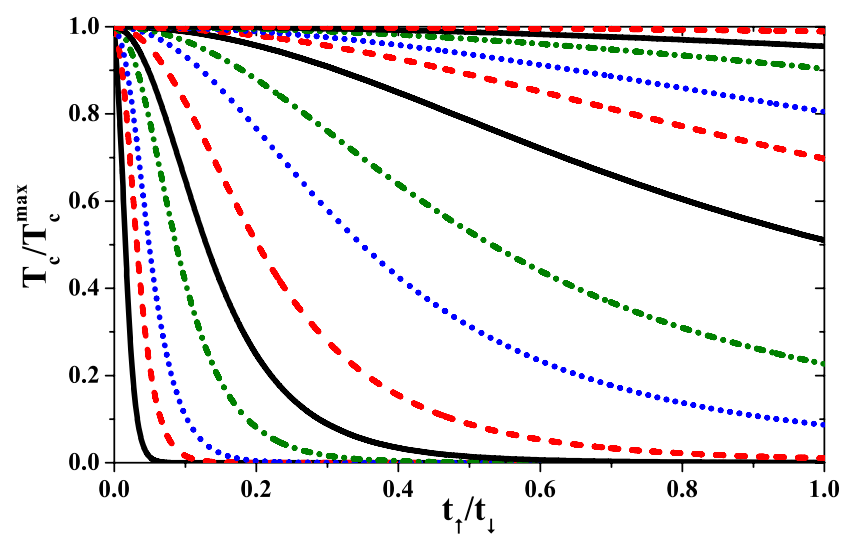

Fig. 4 Temperature of order-disorder transition $T_{c} / T_{c}^{\max }$ as a function of $t_{\uparrow} / t_{\downarrow}$ for different values of $W / t_{\downarrow}$ (from the bottom-left: 0.02, 0.04, $0.06,0.10,0.14,0.20,0.30,0.40,0.60,0.80,1.00,1.40,2.00$, and 4.00). $T_{c}^{\max } \equiv T_{c}\left(t_{\uparrow}=0\right)$ 
Fig. 5 Parameters $\Delta_{Q}$ (a) and $M_{Q}(\mathbf{b})$ as a function of reduced temperature $T / T_{c}$ for $W / t_{\downarrow}=0.4$ and $t_{\uparrow} / t_{\downarrow}=$ $0.00,0.25,0.50,0.75,1.00$ (from the top). For $t_{\uparrow} / t_{\downarrow}=1.00, M_{Q}=0$ at any $T$
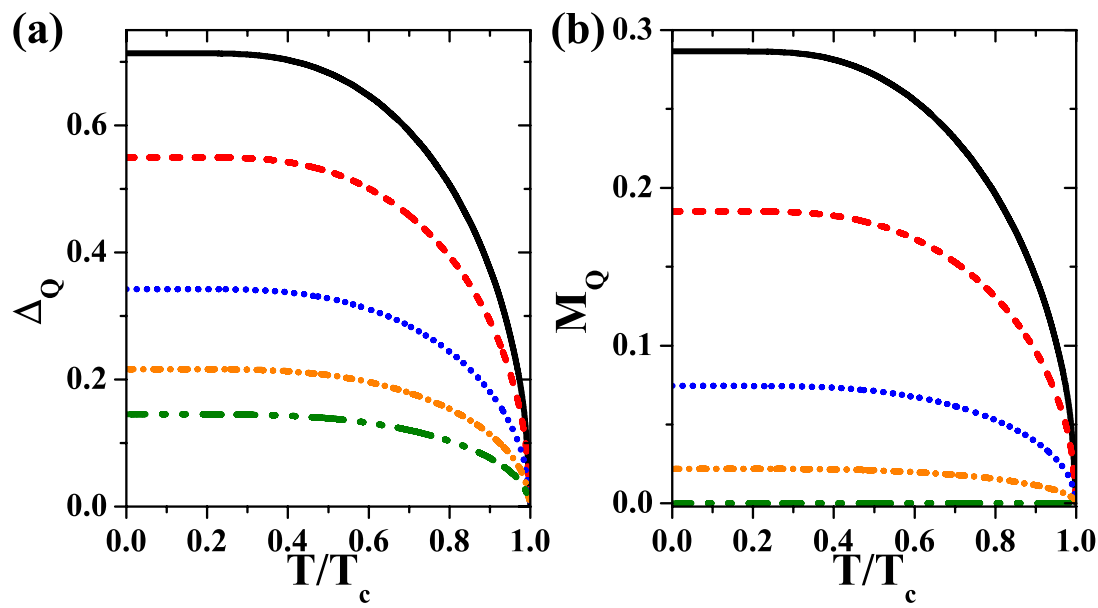

$\Delta_{Q}$ and $M_{Q}$ ((5) and (6), respectively) as a function of $T / T_{c}$ for $W / t_{\downarrow}=0.4$ and a few values of ratio $t_{\uparrow} / t_{\downarrow}$. Note that, only for $t_{\uparrow}=t_{\downarrow}$, parameter $M_{Q}=0$ for any $T$ (in particular, not only for $T>T_{c}$, but also for $T<T_{c}$, where $\Delta_{Q}>0$ ). For other values of $t_{\uparrow} / t_{\downarrow}$ and any finite $W$, both $\Delta_{Q}$ and $M_{Q}$ are nonzero below $T_{c}$. Nevertheless, for $W / t_{\downarrow} \rightarrow+\infty$ and any $t_{\uparrow} / t_{\downarrow}$ parameter $M_{Q} \rightarrow 0$ approaching results for the atomic limit of the extended Hubbard model with $U=0$ $[32,35,36]$.

One should underline that we checked that (5) has only one nonzero solution for $\Delta_{Q}>0$ (if it exists, i.e., for $T<T_{c}$ ) and its corresponding free energy $F\left(\Delta_{Q}\right)$ (calculated from (3) and (4)) is always lower than the energy corresponding to the solution with $\Delta_{Q}=0$ as it should be in the case of the continuous transition. It is equivalent with the statement that free energy $F$ has only one (local) minimum with respect to $\Delta_{Q} \geq 0$. For $T<T_{c}$ energy $F\left(\Delta_{Q}\right)$ has one local maximum for $\Delta_{Q}=0$ and thus the solution with $\Delta_{Q}=0$ is unstable. $\Delta_{Q}$, for which $F\left(\Delta_{Q}\right)$ has a local minimum, is a solution of (5). In Fig. 6, we presents free energy $F$ as a function of $\Delta_{Q}$ for a few different temperatures and an exemplary, but representative,

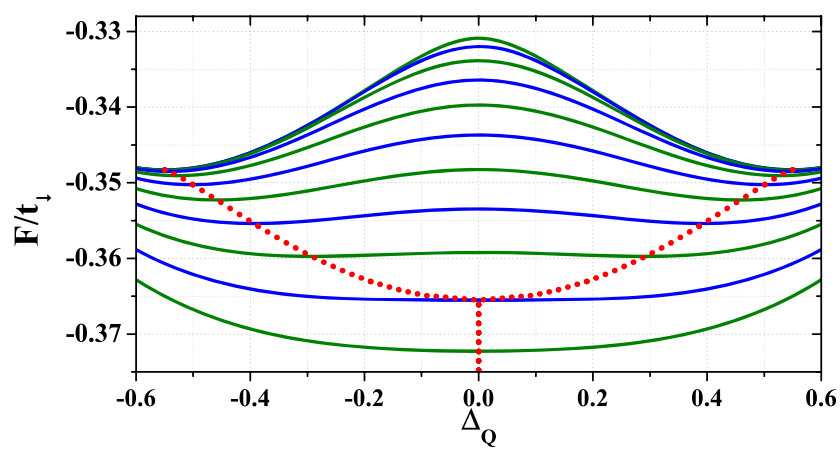

Fig. 6 Free energy $F / t_{\downarrow}$ per site as a function of $\Delta_{Q}$ for $t_{\uparrow} / t_{\downarrow}=0.25$ and $W / t_{\downarrow}=0.4$ for different values of $T / T_{c}$ (from 0.1 to 1.1 with increment 0.1 , from the top to the bottom, solid lines). The dotted line denotes the energies for $\Delta_{Q}$ being the solution of (5) set of the parameters. A minimum at $\Delta_{Q}<0$ for $T<T_{c}$ is a result of the equivalence of both sublattices (in such a case $M_{Q}$ is also negative; $F\left(\Delta_{Q}\right)=F\left(-\Delta_{Q}\right)$, cf. (3) and (4)). In this work, we discussed only $\Delta_{Q} \geq 0$ solutions.

\section{Conclusions and Final Remarks}

In this paper, we investigated the asymmetric model for itinerant spin- $1 / 2$ fermions on the lattice, which can interact with each other only via nearest-neighbor density-density interaction. We obtained its phase diagram at the half-filling as a function of $W / t_{\downarrow}$ and $t_{\uparrow} / t_{\downarrow}$ and found that there is the continuous transition from the ordered phase (with coexisting charge and antiferromagnetic orders) to the nonordered phase at large temperatures. The magnetic order vanishes in the ordered phase only for $t_{\uparrow}=t_{\downarrow}$ or in $W \rightarrow+\infty$ limit. Both, charge-order parameter $\Delta_{Q}$ at $T=0$ and critical temperature $T_{c}$, are decreasing function of $t_{\uparrow} / t_{\downarrow}$ and increases with $W / t_{\downarrow}$.

Note that the dependencies of $T_{c}$ as a function of $W / t_{\downarrow}$ (Fig. 3) and $t_{\uparrow} / t_{\downarrow}$ (Fig. 4) are indeed very similar as those of $\Delta_{Q}$ in the ground state (Figs. 1a and 2a, respectively), but they do not coincide. These similar behaviors are in an agreement with the fact that $W$ interaction is the driving force for an emergence of the charge order, whereas the antiferromagnetic order is due to a difference of the hopping amplitudes for two electron species. Parameters $\Delta_{Q}$ and $M_{Q}$ can define parameters $\Delta_{Q \uparrow}=\Delta_{Q}+M_{Q}$ and $\Delta_{Q \downarrow}=\Delta_{Q}-M_{Q}$, which reflect inhomogeneous particle distribution for each spin (they are differences between concentrations of particles in each sublattice for each spin, separately, cf. [29, 30]). For $t_{\uparrow} \leq t_{\downarrow}$, we found that $\Delta_{Q} \geq$ $M_{Q} \geq 0$ and thus $\Delta_{Q \uparrow} \geq \Delta_{Q \downarrow} \geq 0$. It can be intuitively understood as a result of the fact that, in model (1), spin- $\downarrow$ particles are less localized (more delocalized) than particles with spin- $\uparrow\left(\Delta_{Q \uparrow}>\Delta_{Q \downarrow}\right.$ for $\left.t_{\uparrow}<t_{\downarrow}\right)$. 
The Hartree decoupling of the intersite term used in this work is an exact theory for the model (1) in the case of large coordination number $(z \rightarrow+\infty$ limit) [11-14]. On the contrary, in the case of nonzero onsite interaction (i.e., $U \neq 0$ ), the Hartree-Fock approach is not rigorous and one needs to use more elaborated techniques (such as the dynamical mean-field theory [2-5]) to get some exact results, even for $z \rightarrow+\infty$. Note also that the two-sublattice assumption (i.e., restriction to the checker-board orderings) is justified for the half-filling [39, 40], whereas for other particle concentrations (i.e., $n \neq 1$ ) various stripe and incommensurate orderings could appear, e.g., [24, 25].

Model (1) is rather simple and can be treated as a toy model. But recent development of experimental techniques in ultra-cold atomic gases allows to tune various interactions between particles [6-10]. Because they are setups with fully controllable parameters, the experimental realization of the system described by the Hamiltonian studied in this work (with parameters treated as the effective ones) could be possible [41-44].

Nevertheless, it is worth to mention that the particular case, where one of the two electronic subsystems is close to a Lifshitz electronic topological transition (where the ratio of the Coulomb repulsion energy over the Fermi energy is large) [45-47], has been shown to be a common feature of the high temperature superconductors going through to hydrides [48], diborides [49], iron-based superconductors [50, 51], oxide interfaces [52], and organics [53] as well as cuprates [54, 55]. One should also indicate the fact that electronic correlated systems with one narrow band component are close to a complex phase separation, cf., e.g., Refs. [56-59].

Acknowledgements The authors sincerely thank dr Andrzej Ptok for very fruitful discussions. K. J. K. appreciates also founding in the frame of a scholarship of the Minister of Science and Higher Education (Poland) for outstanding young scientists (2019 edition, no. 821/STYP/14/2019). K. M.-A. acknowledges the hospitality of the Henryk Niewodniczański Institute of Nuclear Physics (of the Polish Academy of Sciences) in Kraków during her student internship.

Funding Information K. J. K. received financial support from the National Science Centre (NCN, Poland) under grant SONATINA no. UMO-2017/24/C/ST3/00276.

Open Access This article is licensed under a Creative Commons Attribution 4.0 International License, which permits use, sharing, adaptation, distribution and reproduction in any medium or format, as long as you give appropriate credit to the original author(s) and the source, provide a link to the Creative Commons licence, and indicate if changes were made. The images or other third party material in this article are included in the article's Creative Commons licence, unless indicated otherwise in a credit line to the material. If material is not included in the article's Creative Commons licence and your intended use is not permitted by statutory regulation or exceeds the permitted use, you will need to obtain permission directly from the copyright holder. To view a copy of this licence, visit http:// creativecommonshorg/licenses/by/4.0/.

\section{References}

1. Micnas, R., Ranninger, J., Robaszkiewicz, S.: Superconductivity in narrow-band systems with local nonretarded attractive interactions. Rev. Mod. Phys. 62, 113 (1990). https://doi.org/10.1103/ RevModPhys.62.113

2. Georges, A., Kotliar, G., Krauth, W., Rozenberg, M.J.: Dynamical mean-field theory of strongly correlated fermion systems and the limit of infinite dimensions. Rev. Mod. Phys. 68, 13 (1996). https://doi.org/10.1103/RevModPhys.68.13

3. Imada, M., Fujimori, A., Tokura, Y.: Metal-insulator transitions. Rev. Mod. Phys. 70, 1039 (1998). https://doi.org/10.1103/ RevModPhys.70.1039

4. Freericks, J.K., Zlatić, V.: Exact dynamical mean-field theory of the Falicov-Kimball model. Rev. Mod. Phys. 75, 1333 (2003). https://doi.org/10.1103/RevModPhys.75.1333

5. Kotliar, G., Savrasov, S.Y., Haule, K., Oudovenko, V.S., Parcollet, O., Marianetti, C.A.: Electronic structure calculations with dynamical mean-field theory. Rev. Mod. Phys. 78, 865 (2006). https://doi.org/10.1103/RevModPhys.78.865

6. Bloch, I., Dalibard, J., Zwerger, W.: Many-body physics with ultracold gases. Rev. Mod. Phys. 80, 885 (2008). https://doi.org/10.1103/RevModPhys.80.885

7. Giorgini, S., Pitaevskii, L.P., Stringari, S.: Theory of ultracold atomic fermi gases. Rev. Mod. Phys. 80, 1215 (2008). https://doi.org/10.1103/RevModPhys.80.1215

8. Guan, X.-W., Batchelor, M.T., Lee, C.: Fermi gases in one dimension: from Bethe ansatz to experiments. Rev. Mod. Phys. 85 , 1633 (2013). https://doi.org/10.1103/RevModPhys.85.1633

9. Georgescu, I.M., Ashhab, S., Nori, F.: Quantum simulation. Rev. Mod. Phys. 86, 153 (2014). https://doi.org/10.1103/RevModPhys.86.

10. Dutta, O., Gajda, M., Hauke, P., Lewenstein, M., Lühmann, D.S., Malomed, B.A., Sowiński, T., Zakrzewski, J.: Non-standard Hubbard models in optical lattices: a review. Rep. Prog. Phys. 78, 066001 (2015). https://doi.org/10.1088/0034-4885/78/6/066001

11. Müller-Hartmann, E.: Correlated fermions on a lattice in high dimensions. Z. Phys. B 74, 507 (1989). https://doi.org/10.1007/ BF01311397

12. Müller-Hartmann, E.: Fermions on a lattice in high dimensions. Int. J. Mod. Phys. B 3, 2169 (1989). https://doi.org/10.1142/ S0217979289001391

13. Metzner, W., Vollhardt, D.: Correlated lattice fermions in $d=\infty$ dimensions. Phys. Rev. Lett. 62, 324 (1989). https://doi.org/10.1103/

14. Metzner, W.: Variational theory for correlated lattice fermions in high dimensions. Z. Phys. B 77, 253 (1989). https://doi.org/10.1007/BF01313669

15. Winograd, E.A., Chitra, R., Rozenberg, M.J.: Orbital-selective crossover and Mott transitions in an asymmetric Hubbard model of cold atoms in optical lattices. Phys. Rev. B 84, 233102 (2011). https://doi.org/10.1103/PhysRevB.84.233102

16. Dao, T.-L., Ferrero, M., Cornaglia, P.S., Capone, M.: Mott transition of fermionic mixtures with mass imbalance in optical lattices. Phys. Rev. A 85, 013606 (2012). https://doi.org/10.1103/PhysRevA.

17. Winograd, E.A., Chitra, R., Rozenberg, M.J.: Phase diagram of the asymmetric Hubbard model and an entropic chromatographic method for cooling cold fermions in optical lattices. Phys. Rev. B 86, 195118 (2012). https://doi.org/10.1103/PhysRevB.86.195118

18. Amaricci, A., Camjayi, A., Haule, K., Kotliar, G., Tanasković, D., Dobrosavljević, V.: Extended Hubbard model: Charge ordering and Wigner-Mott transition. Phys. Rev. B 82, 155102 (2010). https://doi.org/10.1103/PhysRevB.82.155102 
19. Huang, L., Ayral, T., Biermann, S., Werner, P.: Extended dynamical mean-field study of the Hubbard model with long-range interactions. Phys. Rev. B 90, 195114 (2014). https://doi.org/10.1103/ PhysRevB.90.195114

20. Kapcia, K.J., Robaszkiewicz, S., Capone, M., Amaricci, A.: Doping-driven metal-insulator transitions and charge orderings in the extended Hubbard model. Phys. Rev. B 95, 125112 (2017). https://doi.org/10.1103/PhysRevB.95.125112

21. Terletska, H., Chen, T., Paki, J., Gull, E.: Charge ordering and nonlocal correlations in the doped extended Hubbard model. Phys. Rev. B 97, 115117 (2018). https://doi.org/10.1103/PhysRevB.97. 115117

22. Paki, J., Terletska, H., Iskakov, S., Gull, E.: Charge order and antiferromagnetism in the extended Hubbard model. Phys. Rev. B 99, 245146 (2019). https://doi.org/10.1103/PhysRevB.99.245146

23. Pudleiner, P., Kauch, A., Held, K., Li, G.: Competition between antiferromagnetic and charge density wave fluctuations in the extended Hubbard model. Phys. Rev. B 100, 075108 (2019). https://doi.org/10.1103/PhysRevB.100.075108

24. Uhrig, G.S., Vlaming, R.: Inhibition of phase separation and appearance of new phases for interacting spinless fermions. Phys. Rev. Lett. 71, 271 (1993). https://doi.org/10.1103/PhysRevLett.71.

25. Uhrig, G.S., Vlaming, R.: Zero and finite temperature phase diagram of the spinless fermion model in infinite dimensions. Ann. Phys. (Berlin, Ger.) 507, 778 (1995). https://doi.org/10.1002/andp. 19955070805

26. van Dongen, P.G.J., Vollhardt, D.: Exact mean-field hamiltonian for fermionic lattice models in high dimensions. Phys. Rev. Lett. 65, 1663 (1990). https://doi.org/10.1103/PhysRevLett.65.1663

27. van Dongen, P.G.J.: Exact mean-field theory of the extended simplified Hubbard model. Phys. Rev. B 45, 2267 (1992). https://doi.org/10.1103/PhysRevB.45.2267

28. van Dongen, P.G.J., Leinung, C.: Mott-Hubbard transition in a magnetic field. Ann. Phys. (NY) 509, 45 (1997). https://doi.org/10.1002/andp.19975090104

29. Lemański, R., Kapcia, K.J., Robaszkiewicz, S.: Extended FalicovKimball model: exact solution for the ground state. Phys. Rev. B 96, 205102 (2017). https://doi.org/10.1103/PhysRevB.96.205102

30. Kapcia, K.J., Lemański, R., Robaszkiewicz, S.: Extended Falicov-Kimball model: exact solution for finite temperatures. Phys. Rev. B 99, 245143 (2019). https://doi.org/10.1103/PhysRevB.99.245143

31. Farkašovský, P.: The influence of nonlocal interactions on valence transitions and formation of excitonic bound states in the generalized Falicov-Kimball model. Eur. Phys. J. B 92, 141 (2019). https://doi.org/10.1140/epjb/e2019-100051-6

32. Micnas, R., Robaszkiewicz, S., Chao, K.A.: Multicritical behavior of the extended Hubbard model in the zero-bandwidth limit. Phys. Rev. B 29, 2784 (1984). https://doi.org/10.1103/PhysRevB.29.2784

33. Jędrzejewski, J.: Phase diagrams of extended Hubbard models in the atomic limit. Phys. A (Amsterdam) 205, 702 (1994). https://doi.org/10.1016/0378-4371(94)90231-3

34. Borgs, C., Jędrzejewski, J., Kotecký, R.: The staggered chargeorder phase of the extended Hubbard model in the atomic limit. J. Phys. A: Math. Gen. 29, 733 (1996). https://doi.org/10.1088/03054470/29/4/005

35. Kapcia, K., Robaszkiewicz, S.: The effects of next-nearestneighbour density-density interaction in the atomic limit of the extended Hubbard model. J. Phys.: Condens. Matter 23, 105601 (2011). https://doi.org/10.1088/0953-8984/23/10/105601

36. Kapcia, K.J., Robaszkiewicz, S.: On the phase diagram of the extended Hubbard model with intersite density-density interactions in the atomic limit. Phys. A (Amsterdam) 461, 487 (2016). https://doi.org/10.1016/j.physa.2016.05.056
37. Kapcia, K.J., Barański, J., Ptok, A.: Diversity of charge orderings in correlated systems. Phys. Rev. E 96, 042104 (2017). https://doi.org/10.1103/PhysRevE.96.042104

38. Kaneko, R., Nonomura, Y., Kohno, M.: Thermal algebraic-decay charge liquid driven by competing short-range Coulomb repulsion. Phys. Rev. B 97, 205125 (2018). https://doi.org/10.1103/ PhysRevB.97.205125

39. Lieb, E.H.: A model for crystallization: a variation on the Hubbard model. Phys. A (Amsterdam) 140, 240 (1986). https://doi.org/10.1016/0378-4371(86)90228-1

40. Kennedy, T., Lieb, E.H.: An itinerant electron model with crystalline or magnetic long range order. Phys. A (Amsterdam) 138, 320 (1986). https://doi.org/10.1016/0378-4371(86)90188-3

41. Hoang, A.-T., Nguyen, T.-H.-Y., Tran, T.-T.-T., Le, D.-A.: Two-component Fermions in optical lattice with spatially alternating interactions. J. Phys. Soc. Jpn. 85, 104702 (2016). https://doi.org/10.7566/JPSJ.85.104702

42. Ptok, A.: The influence of the dimensionality of the system on the realization of unconventional Fulde-Ferrell-Larkin-Ovchinnikov pairing in ultra-cold Fermi gases. J. Phys. Condens. Matter. 29, 475901 (2017). https://doi.org/10.1088/1361-648X/aa928d

43. Amico, A., Scazza, F., Valtolina, G., Tavares, P.E.S., Ketterle, W., Inguscio, M., Roati, G., Zaccanti, M.: Time-resolved observation of competing attractive and repulsive short-range correlations in strongly interacting Fermi gases. Phys. Rev. Lett. 121, 253602 (2018). https://doi.org/10.1103/PhysRevLett.121.253602

44. Yin, X.Y., Hu, H., Liu, X.-J.: Cluster formation in twocomponent Fermi gases. Phys. Rev. Lett. 123, 073401 (2019). https://doi.org/10.1103/PhysRevLett.123.073401

45. Perali, A., Bianconi, A., Lanzara, A., Saini, N.L.: The gap amplification at a shape resonance in a superlattice of quantum stripes: a mechanism for high $T_{c}$. Solid State Commun. 100, 181 (1996). https://doi.org/10.1016/0038-1098(96)00373-0

46. Valletta, A., Bianconi, A., Perali, A., Saini, N.L.: Electronic and superconducting properties of a superlattice of quantum stripes at the atomic limit. Z. Phys. B 104, 707 (1997). https://doi.org/10.1007/s002570050513

47. Ptok, A., Kapcia, K.J., Cichy, A., Oleś, A.M., Piekarz, P.: Magnetic Lifshitz transition and its consequences in multiband iron-based superconductors. Sci. Rep. 7, 41979 (2017). https://doi.org/10.1038/srep41979

48. Bianconi, A., Jarlborg, T.: Superconductivity above the lowest Earth temperature in pressurized sulfur hydride. EPL (Europhys Lett.) 112, 37001 (2015). https://doi.org/10.1209/0295-5075/112/ 37001

49. Agrestini, S., Metallo, C., Filippi, M., Simonelli, L., Campi, G., Sanipoli, C., Liarokapis, E., De Negri, S., Giovannini, M., Saccone, A., Latini, A., Bianconi, A.: Substitution of Sc for $\mathrm{Mg}$ in $\mathrm{MgB}_{2}$ : effects on transition temperature and Kohn anomaly. Phys. Rev. B 70, 134514 (2004). https://doi.org/10.1103/PhysRevB.70. 134514

50. Innocenti, D., Poccia, N., Ricci, A., Valletta, A., Caprara, S., Perali, A., Bianconi, A.: Resonant and crossover phenomena in a multiband superconductor: tuning the chemical potential near a band edge. Phys. Rev. B 82, 1845 (2010). https://doi.org/10.1103/PhysRevB.82.184528

51. Bianconi, A.: Quantum materials: Shape resonances in superstripes. Nat. Phys. 9, 536 (2013). https://doi.org/10.1038/nphys2738

52. Bianconi, A., Innocenti, D., Valletta, A., Perali, A.: Shape resonances in superconducting gaps in a 2DEG at oxideoxide interface. J. Phys.: Conf. Series 529, 012007 (2014). https://doi.org/10.1088/1742-6596/529/1/012007

53. Mazziotti, M.V., Valletta, A., Campi, G., Innocenti, D., Perali, A., Bianconi, A.: Possible Fano resonance for high- $T_{c}$ multigap superconductivity in $\mathrm{p}$-Terphenyl doped by $\mathrm{K}$ at the 
Lifshitz transition. EPL Europhys Lett. 118, 37003 (2017). https://doi.org/10.1209/0295-5075/118/37003

54. Bianconi, A.: Feshbach shape resonance in multiband superconductivity in heterostructures. J. Supercond. 18, 625 (2005). https://doi.org/10.1007/s10948-005-0047-5

55. Perali, A., Innocenti, D., Valletta, A., Bianconi, A.: Anomalous isotope effect near a 2.5 Lifshitz transition in a multiband multi-condensate superconductor made of a superlattice of stripes. Supercond. Sci. Technol. 25, 124002 (2012). https://doi.org/10.1088/0953-2048/25/12/124002

56. Kagan, M.Y., Bianconi, A.: Fermi-Bose mixtures and BCS-BEC crossover in high-Tc superconductors. Condens. Matter 4, 51 (2019). https://doi.org/10.3390/condmat4020051

57. Kugel, K.I., Rakhmanov, A.L., Sboychakov, A.O., Poccia, N., Bianconi, A.: Model for phase separation controlled by doping and the internal chemical pressure in different cuprate superconductors. Phys. Rev. B 78, 165124 (2008). https://doi.org/10.1103/PhysRevB.78.165124

58. Kugel, K.I., Rakhmanov, A.L., Sboychakov, A.O., Kusmartsev, F.V., Poccia, N., Bianconi, A.: A two-band model for the phase separation induced by the chemical mismatch pressure in different cuprate superconductors. Supercond. Sci. Technol. 22, 014007 (2008). https://doi.org/10.1088/0953-2048/22/1/014007

59. Bianconi, A., Poccia, N., Sboychakov, A.O., Rakhmanov, A.L., Kugel, K.I.: Intrinsic arrested nanoscale phase separation near a topological Lifshitz transition in strongly correlated twoband metals. Supercond. Sci. Technol. 28, 024005 (2015). https://doi.org/10.1088/0953-2048/28/2/024005

Publisher's Note Springer Nature remains neutral with regard to jurisdictional claims in published maps and institutional affiliations. 